\title{
Aortic Disease in the Young: Genetic Aneurysm Syndromes, Connective Tissue Disorders, and Familial Aortic Aneurysms and Dissections
}

\author{
Marcelo Cury, Fernanda Zeidan, and Armando C. Lobato \\ Instituto de Cirurgia Vascular e Endovascular (ICVE), São Paulo, SP, Brazil \\ Correspondence should be addressed to Marcelo Cury; mpcury@globo.com
}

Received 23 July 2012; Revised 13 December 2012; Accepted 18 December 2012

Academic Editor: Erich Minar

Copyright (C) 2013 Marcelo Cury et al. This is an open access article distributed under the Creative Commons Attribution License, which permits unrestricted use, distribution, and reproduction in any medium, provided the original work is properly cited.

\begin{abstract}
There are many genetic syndromes associated with the aortic aneurysmal disease which include Marfan syndrome (MFS), EhlersDanlos syndrome (EDS), Loeys-Dietz syndrome (LDS), familial thoracic aortic aneurysms and dissections (TAAD), bicuspid aortic valve disease (BAV), and autosomal dominant polycystic kidney disease (ADPKD). In the absence of familial history and other clinical findings, the proportion of thoracic and abdominal aortic aneurysms and dissections resulting from a genetic predisposition is still unknown. In this study, we propose the review of the current genetic knowledge in the aortic disease, observing, in the results that the causative genes and molecular pathways involved in the pathophysiology of aortic aneurysm disease remain undiscovered and continue to be an area of intensive research.
\end{abstract}

\section{Introduction}

The aortic aneurysmal disease is defined as focal dilation of the aorta, based on its original diameter. The dilatation must be at least one and one-half times the normal diameter. Thus, generally, a segment with a diameter of greater than $3.5 \mathrm{~cm}$ is considered as an aortic aneurysm.

Thoracic aneurysms are those located above the diaphragm and may involve one or more aortic segments (aortic root, ascending aorta, arch, or descending aorta) and are classified accordingly. When they are located under diaphragm, aneurysms are classified as abdominal. The second ones are much more prevalent than thoracic aortic aneurysms. About $80 \%$ of aortic aneurysms occur between the renal arteries and the aortic bifurcation.

Aortic aneurysm rupture represents the 13th cause of death in the USA, accounting for nearly 15,000 of deaths annually [1]. Although, often a late onset disease, there are a significant proportion of patients with presentation at age less than 60. Cases of aortic aneurysms in children have also been reported; however, they are very rare [2]. In this group, the proportion of thoracic or abdominal aortic aneurysms and dissections resulting from a genetic predisposition is unknown. In the absence of an identifiable genetic syndrome, familial aggregation of these aneurysms is well established. The first-degree relatives of an individual with an aortic aneurysm have a ten to twelvefold increased risk of developing aortic aneurysms [3-5].

There are multiple genetic syndromes associated with aortic aneurysmal disease (Table 1). The Key syndromes to consider in any patient who presents at a young age an aortic aneurysm include Marfan syndrome (MFS), Ehlers-Danlos syndrome (EDS), Loeys-Dietz syndrome (LDS), familial thoracic aortic aneurysms and dissections (TAAD), bicuspid aortic valve (BAV), and autosomal dominant polycystic kidney disease (ADPKD). Less common are aortic aneurysms associated with Turner syndrome, neurofibromatosis, tuberous sclerosis, Noonan syndrome, osteogenesis imperfecta and homocystinuria.

\section{Methods}

A research was conducted of the Medline and Ovid Embase databases from 1966 to present and publications gathered 
using search terms of "Marfan," "Ehlers-Danlos," "LoeysDietz," "Polycystic Kidney," "Aortic Aneurysm," "Genetic," "Aortic Dissection," "Connective Tissue," "Familial." Combinations of the terms above were used to generate a list of publications for this paper, as well as papers and relevant references found in the medical literature data.

\section{Marfan Syndrome (MFS)}

MFS is an autosomal dominant disorder of the connective tissue that is a relatively common disorder affecting 2 to 3 in 10,000 persons [6]. Although it produces the most significant changes in the connective fibers of musculoskeletal, cardiovascular, and ocular systems, it may also affect the pulmonary, nervous, and integumentary fibrils.

The most worrisome abnormalities associated with MFS are those affecting the cardiovascular system. Cardiovascular manifestations are mainly characterized by progressive dilatation of the aortic root, leading to aneurysm formation, which can evolute to an aortic dissection or rupture, if not well conducted and treated. Other established cardiovascular manifestations include mitral valve prolapse, dilatation of the pulmonary artery, and dilatation or dissection of the descending thoracic or abdominal aorta. It is already known that near $80 \%$ of Marfan syndrome morbidity is linked to aortic aneurysm and dissection [7].

The diagnosis of MFS is clinical. Revised clinical diagnostic criteria have been published with significant overlaps between the clinical phenotype of MFS and its connective tissue disorders [8].

Patients suspected of being MFS carriers must be submitted to a complete medical history and clinical examination emphasized on the skeletal, ocular, and cardiovascular systems. The diagnosis of these patients should be made according to Ghent criteria and requires a comprehensive clinical assessment of multiple organ systems (Table 2). Genetic testing can be useful in the diagnosis into selected cases.

Mutations in fibrillin 1 gene (FBN1) account for approximately $70-93 \%$ of patients who meet diagnostic criteria for MFS. FBN1, is a large gene $(110 \mathrm{~kb}, 65$ exons) with more than $>600$ mutations reported until this to date, located on chromosome 15.

Most recent researches associated mutations of the TGFB2 gene as cause of familial thoracic aortic aneurysms and dissections with mild systemic features of Marfan syndrome. Still, further work is needed [9].

\section{Ehlers-Danlos Syndrome (EDS)}

EDS is a group of connective tissue disorders characterized by articular hypermobility, skin extensibility, and tissue fragility (Table 3).

The EDS genes may be inherited via dominant autosomal, recessive autosomal, or $\mathrm{x}$-linked patterns of inheritance [10].

The overall prevalence of EDS is 1 in every 10,000 , depending on the type. Males and females of all racial and ethnic backgrounds have the same prevalence [10-15].
In the past, there were more than 10 recognized types of Ehlers-Danlos syndrome. In 1997, researchers proposed a simpler classification reducing the number of major types to six and gave them descriptive names: the arthrochalasia type, the classic type, the dermatosparaxis type, the hypermobility type, the kyphoscoliosis type, and the vascular type. Other forms of the condition may exist, but they have been reported only in single families or are not well characterized [10].

EDS typing is essential to evaluate the risk of aortic aneurysms. Of all the types, the classical, hypermobile and vascular ones are those who the aortic dilatation is found with a higher prevalence [16-21].

Vascular EDS (formerly EDS type IV) is an autosomal dominant disorder of type III collagen caused by mutations in COL3A1 and occurs in 1 in 100,000 to 1 in 200,000 patients [10-12]. Key clinical features include arterial fragility or rupture, intestinal rupture, uterine rupture during pregnancy, and thin translucent skin. Other findings include, easy bruising and a characteristic facial appearance.

Minor criteria may aid in the diagnosis. If the clinical features are suggestive of vascular EDS, then the analysis of COL $3 A 1$ can detect a mutation in $98-99 \%$ of cases [17].

Observation of structurally abnormal type III collagen from cultured fibroblasts is an alternative method for diagnosis, though it may be less sensitive in identifying vascular EDS. It is a consequence of mutations that decrease the production. Genotype-phenotype correlations are now being determined, improving the answers as far as the knowledge of the genetics studies walks, despite of the early stages of it [18].

Arterial rupture in vascular EDS is most prevalent in the 3rd-4th decade and usually involves mild-sized arteries [15], specially involving thoracic or abdominal vessels.

Frequently, descending and abdominal aorta [19]. The rupture is often spontaneous with no apparent cause. Angiography or arterial puncture is generally contraindicated, although some published cases report a successful endovascular therapies $[20,21]$. Vessel surgical ligation is generally the preferred management in these cases. Any arterial clamping should be performed with extreme caution. Prophylactic surgery is not recommended. Patients can also present intracranial bleeding or stroke. Varicose veins are common in this disorder but should not be treated surgically. Bowel rupture most frequently involves the sigmoid colon.

There is no specific treatment for EDS; however, the diagnosis can help the management of the disease and in genetic counseling.

\section{Loeys-Dietz Syndrome (LDS)}

LDS is a newly described aortic aneurysm syndrome, associated with mutations of the transforming growth factor beta receptor genes 1 and 2 (TGF $\beta R 1 / T G F \beta R 2)$. It is characterized by premature and aggressive aneurysms and dissections, widely spaced in eyes, bifid uvula, or cleft palate, causing generalized arterial tortuosity [22]. It is inherited in a dominant autosomal manner. TGF $\beta R 1 / T G F \beta R 2$ gene mutations lead to an increased signaling of TGF- $\beta$ in blood vessels resulting 
TABLE 1: Aneurysm syndromes.

\begin{tabular}{|c|c|c|}
\hline \multicolumn{3}{|c|}{ Genetic aneurysm syndromes } \\
\hline Marfan syndrome (MFS) & FBN1 & $\begin{array}{l}\text { Dilatation and aneurysm of the aortic root, dilatation of } \\
\text { the pulmonary artery, and dilatation or dissection of } \\
\text { the descending thoracic or abdominal aorta }\end{array}$ \\
\hline Ehlers-Danlos syndrome (EDS) & $\begin{array}{l}\text { COL5A1, COL5A2, and } \\
\text { COL } 3 A 1\end{array}$ & $\begin{array}{l}\text { Arterial mid-sized rupture, specially involving thoracic } \\
\text { or abdominal vessels. Frequently descending and } \\
\text { abdominal aorta [18] }\end{array}$ \\
\hline Loeys-Dietz syndrome (LDS) & TGFBR 1 and TGFBR2 & $\begin{array}{l}\text { Premature and aggressive aneurysms and dissections. } \\
\text { Aneurysms distal to the aortic root }\end{array}$ \\
\hline $\begin{array}{l}\text { Familial aortic aneurysm and/or } \\
\text { dissection syndromes (FAAD) }\end{array}$ & $\begin{array}{l}\text { TGFBR2, MYH11, and } \\
\text { ACTA2 }\end{array}$ & Ascending aorta aneurysm and dissection \\
\hline Bicuspid aortic valve (BAV) & Unknown & $\begin{array}{l}\text { Aortic dilation typically involves the aortic root and } \\
\text { ascending aorta whereas it is not present in the } \\
\text { descending and abdominal aorta }\end{array}$ \\
\hline $\begin{array}{l}\text { Autosomal dominant polycystic } \\
\text { kidney disease (ADPKD) }\end{array}$ & $P K D 1$ and $P K D 2$ & $\begin{array}{l}\text { Dilatation of the aortic root and dissection of the } \\
\text { thoracic aorta }\end{array}$ \\
\hline Turners & $45 \mathrm{X}$ & Thoracic aortic aneurysms and dissections \\
\hline Neurofibromatosis & NF1 & $\begin{array}{l}\text { Aneurysmal arterial disease affecting the abdominal } \\
\text { aorta }\end{array}$ \\
\hline
\end{tabular}

TABLE 2: Major Ghent criteria used to diagnose Marfan syndrome.

\begin{tabular}{|c|c|}
\hline System & Major criteria \\
\hline \multirow{8}{*}{ Skeletal system } & Pectus carinatum \\
\hline & Pectus excavatum requiring surgery \\
\hline & Upper to lower segment ratio $<0.86$ or span to height ratio $>1.05$ \\
\hline & Arachnodactyly: wrist and thumb signs \\
\hline & Pes planus \\
\hline & Protrusio acetabuli \\
\hline & Scoliosis $>20 \mathrm{~d}$ or spondylolisthesis \\
\hline & Reduced extensions at the elbows $(<170 \mathrm{~d})$ \\
\hline Ocular system & Ectopia lentis (dislocated lens) \\
\hline \multirow{2}{*}{ Cardiovascular system } & Dilatation of the ascending aorta \\
\hline & Aortic root dilatation \\
\hline Dura & Lumbosacral dural ectasia \\
\hline \multirow{2}{*}{ Family/genetic history } & Family history \\
\hline & Genetic mutations known to cause Marfan syndrome \\
\hline
\end{tabular}

in overproduction of collagen, loss of elastin, and disarray of elastic fibers. There is a variable clinical expression of the disorder, and to date, clinical diagnostic criteria do not exist [23].

The emerging phenotypes of LDS are divided into two types. Type 1 LDS is a Marfan-like condition associated with severe craniofacial features (craniosynostosis, malar hypoplasia, retrognathia, cleft palate, or abnormal uvula and hypertelorism), aortic root aneurysms, aneurysm of other vessels, arterial tortuosity, arachnodactyly, pectus deformity, scoliosis, joint laxity, and developmental delay.

Type 2 LDS lacks the severe craniofacial features of type 1 LDS. It mimics vascular EDS in the association of a bifid uvula or abnormal palates aortic root aneurysm with dissection and diffuses arterial aneurysms or dissections, arterial tortuosity, and catastrophic complications of pregnancy. Patients do not have cleft palate, hypertelorism, or craniosynostosis.

Overall the median survival of LDS patients is 37 years (in contrast to 48 years for EDS and 70 years for MFS). Mortality is from thoracic or abdominal aortic dissection, rupture, or cerebral hemorrhage. Mean age for the first vascular surgical procedure is about 20 years. The majority will have aneurysms distal to aortic root. Dissection can occur without marked arterial dilatation (in contrast to MFS but similar to EDS). Perioperative vascular surgical mortality is only $1.7 \%$ (in contrast to $45 \%$ for EDS IV). These results prompt 
TABLE 3: Criteria used to diagnose the vascular of EDS.

\begin{tabular}{l}
\hline Major criteria (2 or more highly indicative) \\
Arterial rupture or intestinal, uterine rupture \\
Extensive bruising \\
Thin, translucent skin \\
Characteristic facial appearance \\
\hline Minor criteria \\
Acrogeria \\
Hypermobility of small joints (fingers) \\
Tendon and muscle rupture \\
Clubfoot \\
Varicose veins of early onset \\
Arteriovenous malformation \\
Carotid-cavernous sinus fistula \\
Pneumothorax or hemothorax \\
Positive family history of sudden death \\
\hline
\end{tabular}

early surgical intervention in patients with LDS. Prophylactic repair of adult patients with LDS has been suggested at aortic diameters of $4.0 \mathrm{~cm}$ [24].

Due to the aggressive nature of LDS, genetic testing can be helpful in identifying those patients a great risk of arterial complications, who might be benefited with early surgical intervention. The sensitivity and specificity of $T G F \beta R 1 / T G F \beta R 2$ testing until to date is unknown. Missense mutations in the serine threonine kinase domain of TGF $R$ R1/TGF $\beta R 2$ appear to account for the majority of mutations leading to LDS. There is a reducing penetrance and variable expressivity of them in LDS. Family members testing positive for a TGFBR mutation suggest a higher risk, but not predictive of LDS. Due to the limited number of patients reported to date, the full spectrum of patients with LDS is likely to evolve as additional patients are identified.

\section{Familial Thoracic Aortic Aneurysms and Dissections (TAADs)}

Familial thoracic aortic aneurysm disorder (TAAD) is mostly associated to ascending aorta aneurysm and dissection [25]. Familial aneurysms have been known to occur in described genetic syndromes; however, the genetic basis of nonsyndromic familial aortic aneurysms has only been recently described. These cases demonstrate a familial aggregation of aortic aneurysms. It typically presents itself at a mean age of ten years younger than nonfamilial cases ( 56 years versus 66 years) [26].

Currently, three genes and two loci were identified to be associated with familial TAAD. The first locus to be mapped for familial TAAD was the TAAD1 locus at 5q13-14 [26]. The causative gene at this locus is currently unknown. The second locus to be mapped was the FAA1 locus at 11q23-24, causing more diffuse vascular disease including both thoracic and abdominal aortic aneurysms [27].

The TAAD2 locus was mapped in $3 \mathrm{p} 24-25$, and it is now known that the mutant gene at this locus is TGF $R 2$ [28]. In individuals with $T G F \beta R 2$ mutations, dissection of the aorta may occur before the aorta enlarges to $5.0 \mathrm{~cm}$. The majority of individuals with TAAD resulting from TGF $\beta R 2$ mutations, present initially aortic disease; however, the increased risk for aneurysms and dissections of other vessels includes cerebral aneurysms. The same mutations in TGF $\beta R 2$ have been identified in families with LDS and TAAD, indicating a broad clinical spectrum associated with the same mutations.

The second gene discovered was MYH11. Mutations in MYH11 are associated with familial TAAD as well as patent ductus arteriosus (PDA) [29]. Human MYH11 gene mutations provide the first example of direct changes in a contractile protein produced specifically in smooth muscle cells, leading to an inherited arterial disease.

Recently, a fourth gene was isolated and identified as causing familial TAAD. Missense mutations in ACTA2 were found to be responsible for $14 \%$ of familial TAAD cases [30]. Like MYH11, ACTA2 is also involved in smooth muscle contraction. Aortic pathology from individuals with MYH11 and ACTA2 mutations, revealed evidence of vascular occlusive processes. In some families with ACTA2 mutations, additional evidence of a vascular occlusive process was identified, by the presence of livedo reticularis.

There is also at least one additional major familial aortic aneurysm predisposition locus that remains to be identified.

\section{Bicuspid Aortic Valve (BAV)}

$\mathrm{BAV}$ is among the most common congenital heart malformations, with a prevalence of $1-2 \%$ in the general population [31]. It is associated with serious cardiovascular complications including aortic valve dysfunction, infective endocarditis, aortic dilation, aortic aneurysm, aortic dissection, coarctation of the aorta, interrupted aortic arch, cervicocephalic arterial dissection, and ductus diverticulum aneurysm.

This has led to a hypothesis of a common underlying developmental defect involving the aortic valve and the arterial wall [32]. Up to $50-70 \%$ of such patients with aortic valve dysfunction have evidence of aortic dilation and typically involves the aortic root and ascending aorta, whereas it is not present in the descending and abdominal aorta.

Root dilatation is mostly observed in younger men with $\mathrm{BAV}$ and is unrelated in the presence or severity of any aortic valve stenosis [33]. It is widely known that patients are not protected from subsequent aneurysm formation by aortic valve replacement (AVR), such as implicating inherent abnormalities of the aortic media [34].

Histopathological changes in the ascending aortas of patients with BAVs, including cystic medial necrosis, elastic fiber fragmentation, loss of smooth muscle cells, and changes in their orientation, have been demonstrated [35]. Analysis of the aortic media has revealed less elastic tissue and abnormalities of elastic lamellae in patients with BAV compared to patients with a tricuspid aortic valve [36].

Heritability studies indicate that BAV determination is almost entirely genetic, yet no single BAV gene has been 
identified. However, linkage has been established to chromosomes $18 \mathrm{q}, 5 \mathrm{q}$, and $13 \mathrm{q}$ indicating these regions likely contain genes whose mutation results in BAV [37].

\section{Autosomal Dominant Polycystic Kidney Disease (ADPKD)}

ADPKD is one of the most common genetic disorder, with an estimated prevalence of 1 in 1,000 [37], characterized by progressive cyst development and bilaterally enlarged polycystic kidneys caused by mutations PKD1 ( $85 \%$ of cases) or PKD2 (15\% of cases) [38]. Noncystic abnormalities include intracranial aneurysms and dolichoectasias, dilatation of the aortic root and dissection of the thoracic aorta and cervicocephalic arteries, coronary artery aneurysms, atrial septal aneurysms, mitral valve prolapse, and abdominal wall hernias $[39,40]$.

Cases of aortic dissection in patients with ADPKD demonstrate pathological findings of aortic medial cystic myxoid degeneration and support the central role of primary collagen defect in the pathogenesis of aortic dissection in ADPKD patients [41]. There have been also reported case of ADPKD associated with MFS in an adult [42]. Some cases of patients with ADPKD and abdominal aortic aneurysm (AAA) were related, but there is no definite evidence that the incidence of AAA is affected by ADPKD [43]. Overall ADPKD should be considered a connective tissue disorder regarding similarities to MFS and EDS.

PKD1 and PKD2 are the genes who code the proteins polycystin-1 (PC1) and polycystin-2 (PC2). PC2 forms a nonselective cation channel calcium ion permeable, and PC1 activates and stabilizes that channel. Both are detected in vascular smooth muscle cells (VSMCs) and endothelial cells of all major vessels, including the aorta and intracranial arteries.

The polycystins may be seen as cell-adhesion receptor complexes that link ubiquitous extracellular matrix components to the cell cytoskeleton. Pathologic mutations of PKD1 and PKD2 may disrupt calcium homeostasis and consequently affect transcription of the genes involved in blood vessel structural integrity $[44,45]$. Mutations in PKD1 and $P K D 2$ can cause an increase in both VSMC proliferation and apoptosis. Mouse embryos homozygous for a mutant PKD1 or a $P K D 2$ null allele exhibit a lethal phenotype characterized by the diffuse of vascular ruptures and hemorrhage [44].

\section{Turner Syndrome (TS)}

Turner syndrome is a sex chromosome aneuploidy syndrome with the most frequent chromosome constitution being 45,X, affecting 1 in every 2000 live-born girls [46]. The cardiovascular anomalies include bicuspid aortic valve (BAV), coarctation of the aorta, hypertension, and thoracic aortic aneurysms and dissections. Most of these patients will also demonstrate abnormal aortic arch anatomy [47].

Aortic root dilation is observed in approximately $5 \%$ of Turner patients, and hence routine aortic root surveillance has been suggested. Aortic dissection in TS can occurs with and without associated BAV, as well as coarctation of the aorta or hypertension in aortic diameters of less than $5 \mathrm{~cm}$. Seems to exists a generalized dilatation of major vessels in women with TS, including the aorta, brachial, and carotid arteries [46].

The exact origin of aortic defects in TS remains unknown although histopathology will often demonstrate cystic medial necrosis similar to MFS.

\section{Neurofibromatosis (NF)}

Neurofibromatosis type 1 (NF1) is one of the most common genetic disorders occurring in 1 in 3,000 live births [48]. It is a dominant autosomal disease, which is characterized by café au lait spots, neurofibromas, and Lisch nodules [49].

Approximately half of the NF1 cases occur as spontaneous mutations with no family history of the disorder.

Arterial disease occurs in approximately $10 \%$ of NF1 patients [50]. Aneurysmatic arterial disease affects predominantly the renal arteries and less often the abdominal aorta (middle aortic syndrome) [51] which are the mainly manners.

Aortic disease due to adventitial compression from proliferation of Schwann cells followed by secondary changes of fibrosis and hence presents as stenotic or occlusive disease or due to direct invasion by Schwann cells with intimal thickening and destruction of the media and elastic tissue leading to aneurysm formation is also present [50-53].

\section{Conclusion}

Significant advances have improved the understanding of the association of genetics and aortic aneurismal disease. The disorders discussed above, integrate most of the proportion of the aortic disease, particularly in younger patients. Despite of all these technical improvements, the underlying genetic defects and aberrant molecular pathways remain unknown.

The isolation of new genes and their correlations with genetic syndromes do allow us not only to understand the pathophysiology involved in the genesis of the aortic aneurysm, but also pave the development of better clinical and surgical proposed treatments in affected individuals.

\section{References}

[1] W. S. Moore, "The role of endovascular grafting technique in the treatment of infrarenal abdominal aortic aneurysm," Cardiovascular Surgery, vol. 3, no. 2, pp. 109-114, 1995.

[2] A. V. Sterpetti, W. J. Hunter, and R. D. Schultz, "Congenital abdominal aortic aneurysms in the young. Case report and review of the literature," Journal of Vascular Surgery, vol. 7, no. 6, pp. 763-769, 1988.

[3] P. P. Majumder, P. L. S. Jean, R. E. Ferrell, M. W. Webster, and D. L. Steed, "On the inheritance of abdominal aortic aneurysm," American Journal of Human Genetics, vol. 48, no. 1, pp. 164-170, 1991.

[4] R. M. Sandford, M. J. Bown, N. J. London, and R. D. Sayers, "The genetic basis of abdominal aortic aneurysms: a review," 
European Journal of Vascular and Endovascular Surgery, vol. 33, no. 4, pp. 381-390, 2007.

[5] M. W. Webster, P. L. S. Jean, D. L. Steed, R. E. Ferrell, and P. P. Majumder, "Abdominal aortic aneurysm: results of a family study," Journal of Vascular Surgery, vol. 13, no. 3, pp. 366-372, 1991.

[6] K. K. Singh, K. Rommel, A. Mishra et al., "TGFBR1 and TGFBR2 mutations in patients with features of Marfan syndrome and Loeys-Dietz syndrome," Human Mutation, vol. 27, no. 8, pp. 770-777, 2006.

[7] M. C. Porciani, M. Attanasio, V. Lepri et al., "Prevalence of cardiovascularmanifestations in Marfan syndrome," Italian Heart Journal, vol. 5, no. 8, pp. 647-652, 2004.

[8] A. De Paepe, R. B. Devereux, H. C. Dietz, R. C. Hennekam, and R. E. Pyeritz, "Revised diagnostic criteria for the Marfan syndrome," American Journal of Medical Genetics, vol. 62, no. 4, pp. 417-426, 1996.

[9] C. Boileau, D. C. Guo, N. Hanna, E. S. Regalado, D. Detaint, L. Gong et al., "TGFB2 mutations cause familial thoracic aortic aneurysms and dissections associated with mild systemic features of Marfan syndrome," Nature Genetics, vol. 44, no. 8, pp. 916-921.

[10] P. Beighton, A. De Paepe, B. Steinmann, P. Tsipouras, and R. J. Wenstrup, "Ehlers-Danlos syndromes: revised nosology, Villefranche, 1997. Ehlers-Danlos National Foundation (USA) and Ehlers-Danlos Support Group (UK)," Journal of Medical Genetics, vol. 77, no. 1, pp. 31-37, 1998.

[11] E. J. Lawrence, "The clinical presentation of Ehlers-Danlos syndrome," Advances in Neonatal Care, vol. 5, no. 6, pp. 301-314, 2005.

[12] S. E. Whitelaw, "Ehlers-Danlos Syndrome, classical type: case management," Pediatric Nursing, vol. 29, no. 6, pp. 423-426, 2003.

[13] "What is Ehlers-Danlos syndrome? Ehlers-Danlos National Foundation website," 2008, http://www.ednf.org/.

[14] "Genetics home reference: Ehlers-Danlos syndrome. National Library of Medicine," 2008, http://www.ghr.mlm.nih.gov/.

[15] D. P. Germain and Y. Herrera-Guzman, "Vascular EhlersDanlos syndrome," Annales de Genetique, vol. 47, no. 1, pp. 1-9, 2004.

[16] R. J. Wenstrup, R. A. Meyer, J. S. Lyle et al., "Prevalence of aortic root dilation in the Ehlers-Danlos syndrome," Genetics in Medicine, vol. 4, no. 3, pp. 112-117, 2002.

[17] U. Schwarze, W. I. Schievink, E. Petty et al., "Haploinsufficiency for one COL3A1 allele of type III procollagen results in a phenotype similar to the vascular form of Ehlers-Danlos syndrome, Ehlers-Danlos syndrome type IV,' American Journal of Human Genetics, vol. 69, no. 5, pp. 989-1001, 2001.

[18] A. V. Persikov, R. J. Pillitteri, P. Amin, U. Schwarze, P. H. Byers, and B. Brodsky, "Stability related bias in residues replacing glycines within the collagen triple helix (Gly-Xaa-Yaa) in inherited connective tissue disorders," Human Mutation, vol. 24, no. 4, pp. 330-337, 2004.

[19] M. Pepin, U. Schwarze, A. Superti-Furga, and P. H. Byers, "Clinical and genetic features of Ehlers-Danlos syndrome type IV, the vascular type," New England Journal of Medicine, vol. 342, no. 10, pp. 673-680, 2000.

[20] A. Kurata, H. Oka, T. Ohmomo et al., "Successful stent placement for cervical artery dissection associated with the EhlersDanlos syndrome: case report and review of the literature," Journal of Neurosurgery, vol. 99, no. 6, pp. 1077-1081, 2003.
[21] Y. Sugawara, K. Ban, K. Imai et al., "Successful coil embolization for spontaneous arterial rupture in association with EhlersDanlos syndrome type IV: report of a case," Surgery Today, vol. 34, no. 1, pp. 94-96, 2004.

[22] B. L. Loeys, J. Chen, E. R. Neptune et al., "A syndrome of altered cardiovascular, craniofacial, neurocognitive and skeletal development caused by mutations in TGFBR1 or TGFBR2," Nature Genetics, vol. 37, no. 3, pp. 275-281, 2005.

[23] B. L. Loeys, U. Schwarze, T. Holm et al., "Aneurysm syndromes caused by mutations in the TGF- $\beta$ receptor," The New England Journal of Medicine, vol. 355, no. 8, pp. 788-798, 2006.

[24] J. A. Williams, B. L. Loeys, L. U. Nwakanma et al., "Early surgical experience with Loeys-Dietz: a new syndrome of aggressive thoracic aortic aneurysm disease," Annals of Thoracic Surgery, vol. 83, no. 2, pp. S757-S763, 2007.

[25] M. A. Coady, R. R. Davies, M. Roberts et al., "Familial patterns of thoracic aortic aneurysms," Archives of Surgery, vol. 134, no. 4, pp. 361-367, 1999.

[26] L. Bart, B. L. Loeys, and C. H. Dietz, "Loeys-Dietz syndrome. Gene reviews," 2008, http://www.ncbi.nlm.nih.gov/ books/NBK1133.

[27] D. Guo, S. Hasham, S. Q. Kuang et al., "Familial thoracic aortic aneurysms and dissections genetic: heterogeneity with a major locus mapping to 5q13-14," Circulation, vol. 103, no. 20, pp. 2461-2468, 2001.

[28] C. J. Vaughan, M. Casey, J. He et al., "Identification of a chromosome 11q23.2-q24 locus for familial aortic aneurysm disease, a genetically heterogeneous disorder," Circulation, vol. 103, no. 20, pp. 2469-2475, 2001.

[29] H. Pannu, N. Avidan, V. Tran-Fadulu, and D. M. Milewicz, "Genetic basis of thoracic aortic aneurysms and dissections: potential relevance to abdominal aortic aneurysms," Annals of the New York Academy of Sciences, vol. 1085, pp. 242-255, 2006.

[30] L. Zhu, R. Vranckx, P. K. Van Kien et al., "Mutations in myosin heavy chain 11 cause a syndrome associating thoracic aortic aneurysm/aortic dissection and patent ductus arteriosus," Nature Genetics, vol. 38, no. 3, pp. 343-349, 2006.

[31] D. C. Guo, H. Pannu, V. Tran-Fadulu, C. L. Papke, R. K. Yu, N. Avidan et al., "Mutations in smooth muscle alpha-actin (ACTA2) lead to thoracic aortic aneurysms and dissections," Nature Genetics, vol. 39, no. 12, pp. 1488-1493, 2007.

[32] J. M. Alegret, I. Duran, O. Palazón et al., "Prevalence of and predictors of bicuspid aortic valves in patients with dilated aortic roots," American Journal of Cardiology, vol. 91, no. 5, pp. 619-622, 2003.

[33] M. Cecconi, S. Nistri, A. Quarti et al., "Aortic dilatation in patients with bicuspid aortic valve," Journal of Cardiovascular Medicine, vol. 7, no. 1, pp. 11-20, 2006.

[34] C. A. Della, C. Bancone, C. Quarto et al., "Predictors of ascending aortic dilatation with bicuspid aortic valve: a wide spectrum of disease expression," European Journal of Cardiothoracic Surgery, vol. 31, no. 3, pp. 397-405, 2007.

[35] T. G. Gleason, "Heritable disorders predisposing to aortic dissection," Seminars in Thoracic and Cardiovascular Surgery, vol. 17, no. 3, pp. 274-281, 2005.

[36] K. Niwa, J. K. Perloff, S. M. Bhuta et al., "Structural abnormalities of great arterial walls in congenital heart disease: light and electron microscopic analyses," Circulation, vol. 103, no. 3, pp. 393-400, 2001.

[37] M. Bauer, M. Pasic, R. Meyer et al., "Morphometric analysis of aortic media in patients with bicuspid and tricuspid aortic valve," Annals of Thoracic Surgery, vol. 74, no. 1, pp. 58-62, 2002. 
[38] L. J. Martin, V. Ramachandran, L. H. Cripe et al., "Evidence in favor of linkage to human chromosomal regions 18q, 5q and $13 \mathrm{q}$ for bicuspid aortic valve and associated cardiovascular malformations," Human Genetics, vol. 121, no. 2, pp. 275-284, 2007.

[39] N. Hateboer, M. A. V. Dijk, N. Bogdanova et al., "Comparison of phonotypes of polycystic kidney disease types 1 and 2," The Lancet, vol. 353, no. 9147, pp. 103-107, 1999.

[40] E. A. Romão, M. Moysés Neto, S. R. Teixeira, V. F. Muglia, O. M. Vieira-Neto, and M. Dantas, "Renal and extrarenal manifestations of autosomal dominant polycystic kidney disease," Brazilian Journal of Medical and Biological Research, vol. 39, no. 4, pp. 533-538, 2006.

[41] E. Tahvanainen, P. Tahvanainen, H. Kääriäinen, and K. Höckerstedt, "Polycystic liver and kidney diseases," Annals of Medicine, vol. 37, no. 8, pp. 546-555, 2005.

[42] C. C. Lee, W. T. Chang, C. C. Fang, I. L. Tsai, and W. J. Chen, "Sudden death caused by dissecting thoracic aortic aneurysm in a patient with autosomal dominant polycystic kidney disease," Resuscitation, vol. 63, no. 1, pp. 93-96, 2004.

[43] C. W. Biermann and G. Rutishauser, "Polycystic kidneys associated with Marfan syndrome in an adult," Scandinavian Journal of Urology and Nephrology, vol. 28, no. 3, pp. 295-296, 1994.

[44] R. Vanmaele, M. Witbreuk, M. De Broe, P. Van Schil, and R. Lins, "Abdominal aortic aneurysm and polycystic kidneys [12]," Nephron, vol. 69, no. 1, pp. 107-108, 1995.

[45] D. Bichet, D. Peters, A. J. Patel, P. Delmas, and E. Honoré, "Cardiovascular polycystins: insights from autosomal dominant polycystic kidney disease and transgenic animal models," Trends in Cardiovascular Medicine, vol. 16, no. 8, pp. 292-298, 2006.

[46] V. E. Torres, Y. Cai, X. Chen et al., "Vascular expression of polycystin-2," Journal of the American Society of Nephrology, vol. 12, no. 1, pp. 1-9, 2001.

[47] L. A. Matura, V. B. Ho, D. R. Rosing, and C. A. Bondy, "Aortic dilatation and dissection in Turner syndrome," Circulation, vol. 116, no. 15, pp. 1663-1670, 2007.

[48] V. B. Ho, V. K. Bakalov, M. Cooley et al., "Major vascular anomalies in Turner syndrome: prevalence and magnetic resonance angiographic features," Circulation, vol. 110, no. 12, pp. 1694-1700, 2004.

[49] K. Seymour-Dempsey and R. J. Andrassy, "Neurofibromatosis: implications for the general surgeon," Journal of the American College of Surgeons, vol. 195, no. 4, pp. 553-563, 2002.

[50] D. H. Gutmann, A. Aylsworth, J. C. Carey et al., "The diagnostic evaluation and multidisciplinary management of neurofibromatosis 1 and neurofibromatosis 2," Journal of the American Medical Association, vol. 278, no. 1, pp. 51-57, 1997.

[51] Y. P. Cho, G. H. Kang, S. J. Choi, H. Herr, M. S. Han, H. J. Jang et al., "Aneurysm of the popliteal artery in neurofibromatosis," Annals of Vascular Surgery, vol. 19, no. 536, pp. 900-903, 2005.

[52] J. L. Huffman, V. Gahtan, V. D. Bowers, and J. L. Mills, "Neurofibromatosis and arterial aneurysms," American Surgeon, vol. 62, no. 4, pp. 311-314, 1996.

[53] S. Saitoh and S. Matsuda, "Aneurysm of the major vessels in neurofibromatosis," Archives of Orthopaedic and Trauma Surgery, vol. 117, no. 1-2, pp. 110-113, 1998. 


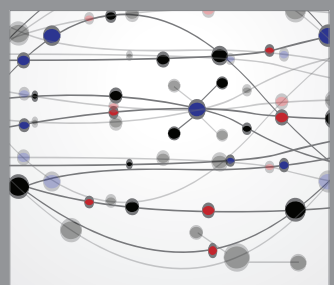

The Scientific World Journal
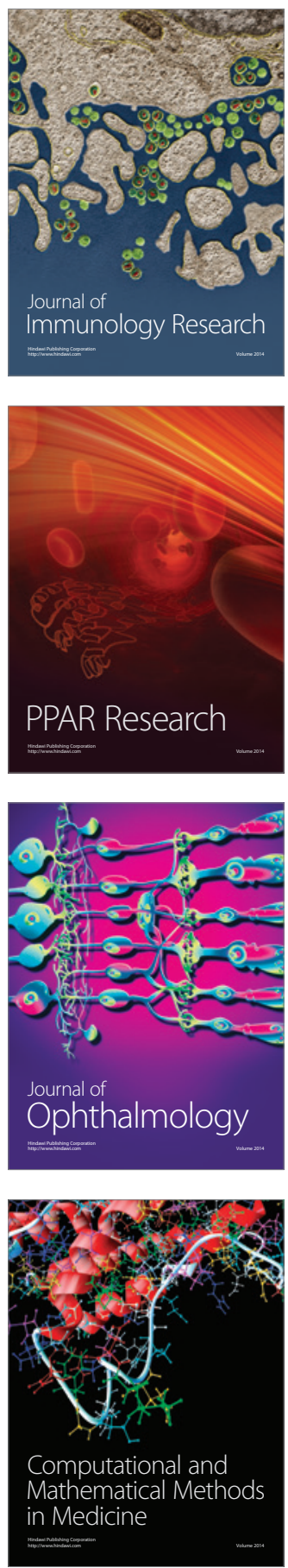

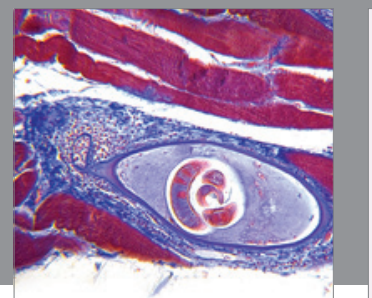

Gastroenterology

Research and Practice
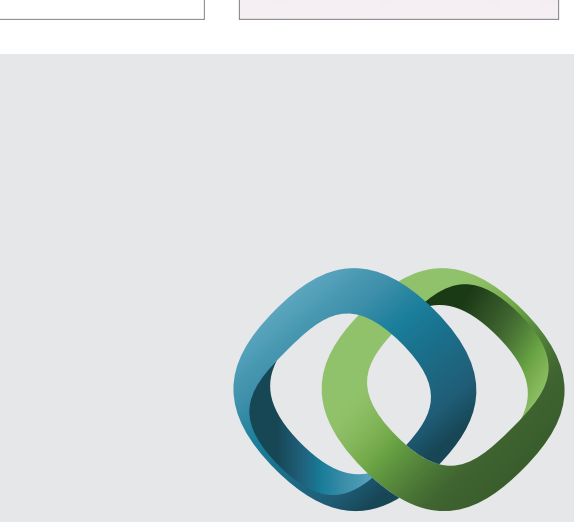

\section{Hindawi}

Submit your manuscripts at

http://www.hindawi.com
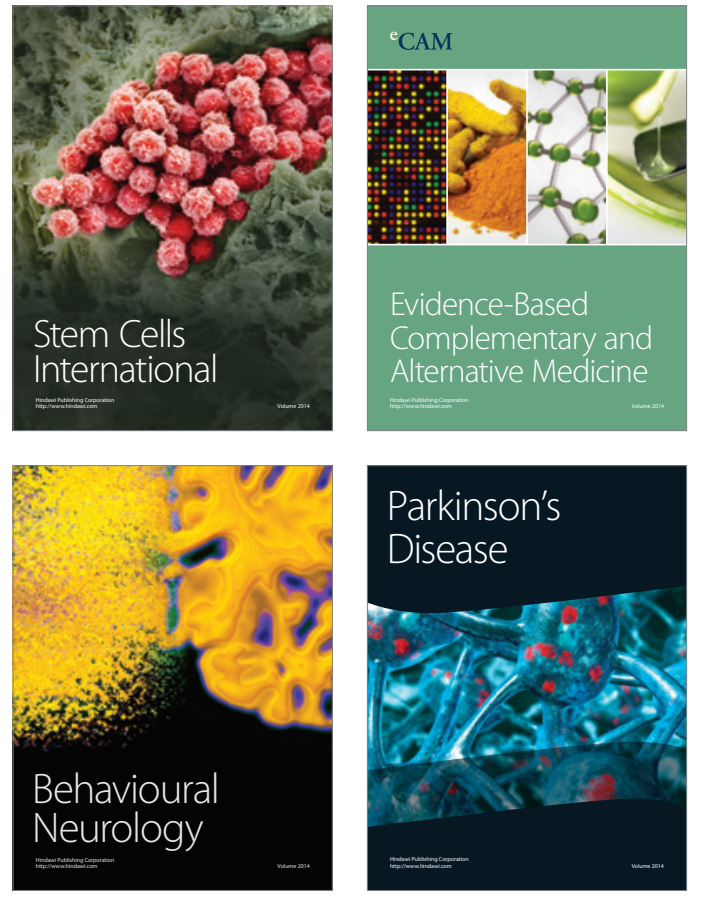
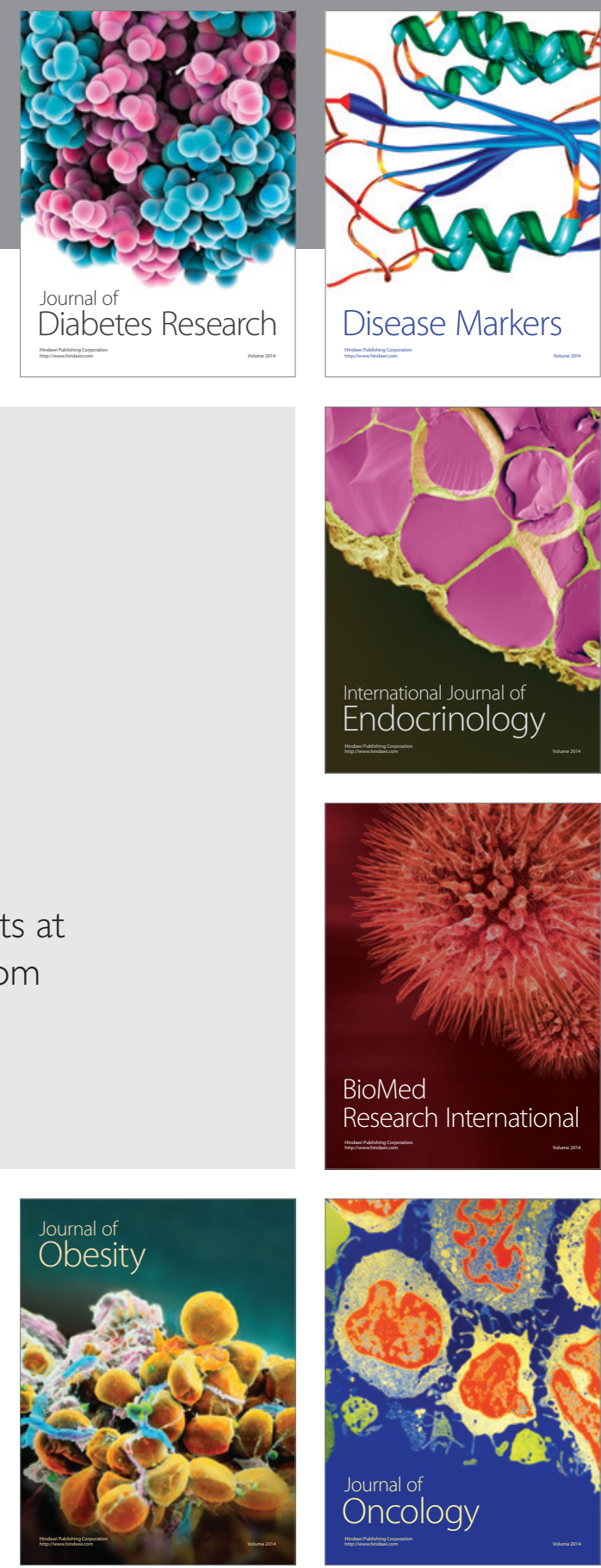

Disease Markers
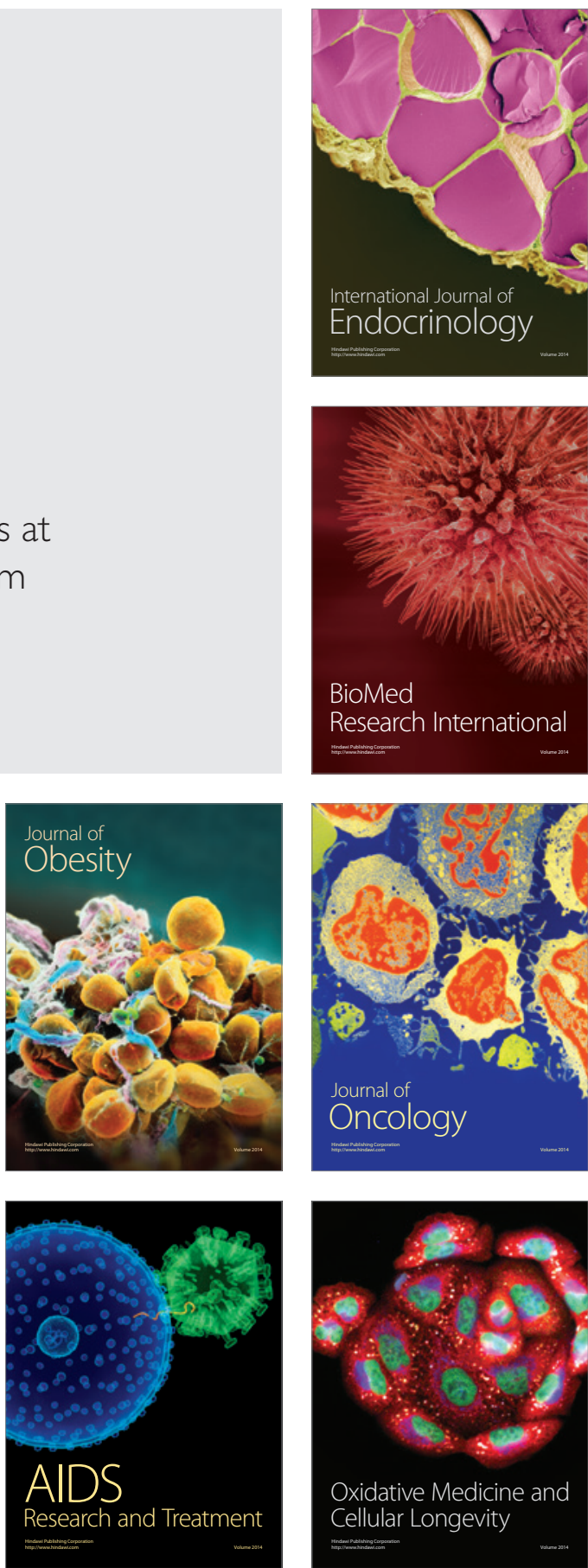